\title{
Exome sequencing reveals a novel MFN2 missense mutation in a Chinese family with Charcot-Marie-Tooth type 2A
}

\author{
YI YOU $^{1 *}$, XIAODONG WANG ${ }^{2 *}$, SHAN LI $^{1}$, XIULI ZHAO ${ }^{1}$ and XUE ZHANG ${ }^{1}$ \\ ${ }^{1}$ Department of Medical Genetics, Institute of Basic Medical Sciences, Chinese Academy of Medical Sciences and \\ Peking Union Medical College, Beijing 100005; ${ }^{2}$ Department of Paediatric Orthopaedics, \\ The Children's Hospital of Soochow University, Suzhou, Jiangsu 215000, P.R. China
}

Received February 28, 2018; Accepted June 8, 2018

DOI: $10.3892 /$ etm.2018.6513

\begin{abstract}
Charcot-Marie-Tooth (CMT) is a group of inherited peripheral neuropathies. To date, mutations in $>80$ genes are reportedly associated with CMT. Protein mitofusin 2 encoded by $M F N 2$ serves an essential role in mitochondrial fusion and regulation of apoptosis, which has previously been reported to be highly associated with an axonal form of neuropathy (CMT2A). In the present study, a large Chinese family with severe CMT was reported and a genetic analysis of the disease was performed. A detailed physical examination for CMT was performed in 13 family members and electrophysiological examinations were performed in 3 affected family members. Whole-exome sequencing was performed on the proband, and the suspected variants were identified by Sanger sequencing. The pathogenicity of mutation was verified by restriction fragment length polymorphism analysis in the family followed by a bioinformatics analysis. A novel c.1190G >C; p.(R397P) mutation in the MFN2 gene was identified in the proband, and co-segregated between genotype and phenotype in the family. The substituted amino acid changed the hydrophobicity and charge characteristics of the mitofusin 2 coiled-coiled domain; thus it may affect its biological function. In summary, a novel pathogenic mutation was identified in a Chinese family with CMT, which expands the phenotypic and mutational spectrum of CMT2A, and provides evidence for prenatal interventions and more precise pharmacological treatments to this family.
\end{abstract}

Correspondence to: Dr Xiuli Zhao or Dr Xue Zhang, Department of Medical Genetics, Institute of Basic Medical Sciences, Chinese Academy of Medical Sciences and Peking Union Medical College, 5 Dongdan San Tiao, Beijing 100005, P.R. China

E-mail: xiulizhao@ibms.pumc.edu.cn

E-mail: xuezhang@pumc.edu.cn

${ }^{*}$ Contributed equally

Key words: Charcot-Marie-Tooth 2A, MFN2, exome sequencing, restriction fragment length polymorphism, bioinformatics analysis

\section{Introduction}

Charcot-Marie-Tooth (CMT) neuropathies, a group of peroneal progressive muscular atrophy disorders, comprise a clinically and genetically heterogeneous group of monogenic disorders affecting the peripheral nerves (1). More than 80 different genes are genetically associated with CMT, leading to a prominent upgrade in diagnostics and understanding of the intricate pathophysiological mechanisms (2). CMT has been categorized into two subsets following neuropathological and electrophysiological criteria: The demyelinating type (CMT1), with slow nerve conduction velocities (NCVs), and the axonal type (CMT2), with milder NCVs (3). The abnormalities in the axons of the peripheral nerves induce hypotonia, foot deformities, distal weakness, sensory loss, and optic atrophy (4).

CMT neuropathies frequently occur in the general population with an estimated prevalence of 1 in 3,000 (2). CMT2 is divided into $\geq 20$ subtypes according to the disease-causing genes, including CMT2A, CMT2B and CMT2D (5). Of these, CMT2A is the most frequent axonal form of CMT allowing for $20-30 \%$ of diagnosed cases. Mutations in kinesin family member 1B (KIF1B; NM_015074) and mitofusin 2 (MFN2) are associated with CMT2A. In contrast to the rare KIF1B gene mutation that causes CMT2A1 (OMIM 118210), different forms of pathogenic mutations in MFN2 most commonly account for autosomal dominant CMT2A2A (OMIM 609260) and autosomal recessive CMT2A2B (OMIM 617087) with differing severity (6). To date, over 170 missense mutations and 20 structural variations has been identified in $M F N 2$, but the mutational mechanism of variation remains unclear. Defected mitofusin 2 can cause early onset severe forms or additional optic atrophy symptoms and accounts for $18 \%$ of families with CMT2 in mainland China (7).

In the present study, the phenotypes of affected members were analyzed in a four generational pedigree and then whole-exome sequencing (WES) was performed on the proband to screen for the causative mutation. The pathogenicity of this mutation was also verified.

\section{Patients and methods}

Patients. A four-generation family from Yancheng (China) with autosomal dominant CMT was recruited in April 2007 
at the Department of Paediatric Orthopaedics, The Children's Hospital of Soochow University (Suzhou, China) (Fig. 1). Blood samples were obtained from 7 affected individuals (3 females and 4 males) with CMT and 6 unaffected family members (II1, II4, II5, II8, II9 and III1) after obtaining written consent. The clinical features of the proband (III2) were analyzed retrospectively. A detailed physical examination was performed on all 7 patients. An electrophysiological examination was available for 3 patients (III3, III4 and IV1). Written informed consent was obtained from all participants and approval was obtained from the Ethics Committee of the Chinese Academy of Medical Sciences (Beijing, China).

WES. Genomic DNA was extracted from peripheral blood of an affected family member (III2) using the QIAamp DNA Blood Mini kit (Qiagen GmbH, Hilden, Germany). The DNA was broken into fragments ranging from 180-280 bp using an ultrasonoscope (S2; Covaris, Inc., Woburn, MA, USA) (8). Adapter oligonucleotides from Illumina (single reads; Illumina Inc., San Diego, CA, USA) were ligated to the ends and fragments were amplified with the Paired-End Sequencing Library Prep kit (Agilent Technologies, Inc., Santa Clara, CA, USA) according to the manufacturer's protocol. Enrichment of coding exons was performed with SureSelectXT Human All Exon V4 (Agilent Technologies, Inc.). After hybridization of the sequencing primer, the bases were incorporated using the Illumina HiSeq2000 platform (Illumina, Inc.) for 90 cycles of sequencing per read to generate paired-end reads including $90 \mathrm{bp}$ at each end and $8 \mathrm{bp}$ of the index tag (9). Image analysis and base calling were performed using Illumina Pipeline (version 1.3.4; Illumina, Inc.).

Error assessment and base calling were also performed using Illumina Pipeline (version 1.3.4) to generate the primary data. Clean reads of 90 bp length were mapped to the GRCh37 reference human genome from the National Center for Biotechnology Information database (NCBI; https://www.ncbi.nlm.nih.gov/) using the Burrows Wheeler Aligner Multi-Vision software package (version, 0.6.2; Wellcome Sanger Institute, Cambridge, UK) (10). Single nucleotide variations (SNVs) and indels were identified using SOAPsnp software (version, 2.04; Beijing Genomics Institute, Shenzhen, China) and GATK Indel Genotyper (version, 3.4-46; http://www.broadinstitute.org/gsa/wiki/index.php/), respectively. Previously identified SNVs were filtered through the NCBI dbVar (https://www.ncbi.nlm.nih.gov/dbvar/browse/) and ExAC browser (http://exac.broadinstitute.org/). Candidate disease-associated genes were obtained using Phenolyzer software (http://phenolyzer.wglab.org/). The OMIM database (https://omim.org) was searched using 'hereditary motor and sensory neuropathy' as key words and the phenotypic series 'Charcot-Marie-Tooth disease-PS118220' (https://omim. org/phenotypicSeries/PS118220). Known disease-causing mutations were recorded in the Human Gene Mutation Database (HGMD) at the Institute of Medical Genetics in Cardiff, UK (http://www.ghmd.cf.ac.uk/).

Candidate mutation confirmed by Sanger sequencing. The genomic DNA of the family members was isolated from peripheral blood using a standard SDS-proteinase K-phenol/chloroform method (11). The reference sequence of the candidate gene MFN2 was obtained from the UCSC Genome Browser (http://genome.ucsc.edu), and the candidate variant was confirmed by PCR-Sanger DNA sequencing. The PCR primers (forward, 5'-TGCTCCTCTGCTTAGTCA-3'; reverse, 5'-GAAACTGGCTGATCAAACGC-3') were designed with Primer 3.0 software (http://primer3.ut.ee/). The PCR reaction contained 20-100 ng genomic DNA, $0.5 \mu$ each primer $(10 \mu \mathrm{M}), 4 \mu \mathrm{l}$ dNTP $(2.5 \mathrm{mM}), 12.5 \mu \mathrm{l} 2 \mathrm{X}$ GC buffer I, 2.5 U LA Taq DNA polymerase (Takara Biotechnology Co., Ltd., Dalian, China) and deionized water to $25 \mu 1$. The PCR program was performed using the following conditions: $95^{\circ} \mathrm{C}$ for $3 \mathrm{~min}$; followed by 38 cycles at $94^{\circ} \mathrm{C}$ for $30 \mathrm{sec}, 58-60^{\circ} \mathrm{C}$ for $30 \mathrm{sec} ; 72^{\circ} \mathrm{C}$ for $50 \mathrm{sec}$; and a final extension at $72^{\circ} \mathrm{C}$ for $8 \mathrm{~min}$. The amplicons were run on an ABI 3730 Sequence Detection System (Applied Biosystems; Thermo Fisher Scientific, Inc., Waltham, MA, USA). The sequencing data were analyzed using CodonCode Aligner (version 6.0.2.6; CodonCode Corporation, Centerville, MA, USA).

Pathogenic validation by restriction fragment length polymorphism analysis. PCR-restriction fragment length polymorphism (PCR-RFLP) analysis was used to verify the candidate mutation in all individuals of the pedigree and 120 healthy Chinese controls (60 males and 60 females; age, 20-45 years) recruited between April 2016 and January 2018. Controls were from the Chinese Han population with no family history of muscle diseases. The first PCR was amplified longer fragments around the mutated site using the procedure stated above. Nested-PCR was performed using a forward (5'-TGC TCCTCTGCTTAGTCA-3') and a mismatched reverse primer 5'-AATTTCAGTCGGTCTTCC-3') using the thermocycling conditions stated above. Amplicons from the nested-PCR were digested at $37^{\circ} \mathrm{C}$ for $15 \mathrm{~min}$ by $M s p \mathrm{I}$ (Takara Biotechnology Co., Ltd.). An $8 \%$ neutral polyacrylamide gel was used to distinguish alleles of the wild type and mutant type by loss or gain of the restriction site. Silver-staining $\left(0.1 \% \mathrm{AgNO}_{3}\right)$ was used at room temperature for $10 \mathrm{~min}$ prior to the final step of the chromogenic reaction.

Pathogenic validation by bioinformatics analysis. The protein sequences of human mitofusin 2 (NP_001121132.1) encoded by MFN2 and of the other homologous proteins from nine different animals were obtained from the NCBI Protein database (https://www.ncbi.nlm.nih.gov/protein/) in FASTA format. Multiple sequence alignment and conservative analyses were performed using MEGA software (version 7; Institute for Genomics and Evolutionary Medicine, Temple University, Philadelphia, PA, USA). The pathogenicity of several missense variants at the same location including that found in the participant family were respectively predicted using online tools PolyPhen-2 (http:/genetics.bwh.harvard. edu/pph2/), Scale-Invariant Feature Transform (SIFT; http://sift.jcvi.org/), MutationTaster (http://www.mutationtaster.org/) and M-CAP (http://bejerano.stanford.edu/mcap/). The three-dimensional structures of normal and mutant mitofusin 2 were generated by homology modeling using SWISS-MODEL (http://swissmodel.expasy.org/) (12). The structure of mitofusin 2 was examined with the crystal structure of the truncated mitofusin 1 structure (pdb 5GOM). The interactions between the mutant amino acid (R397P) and the 


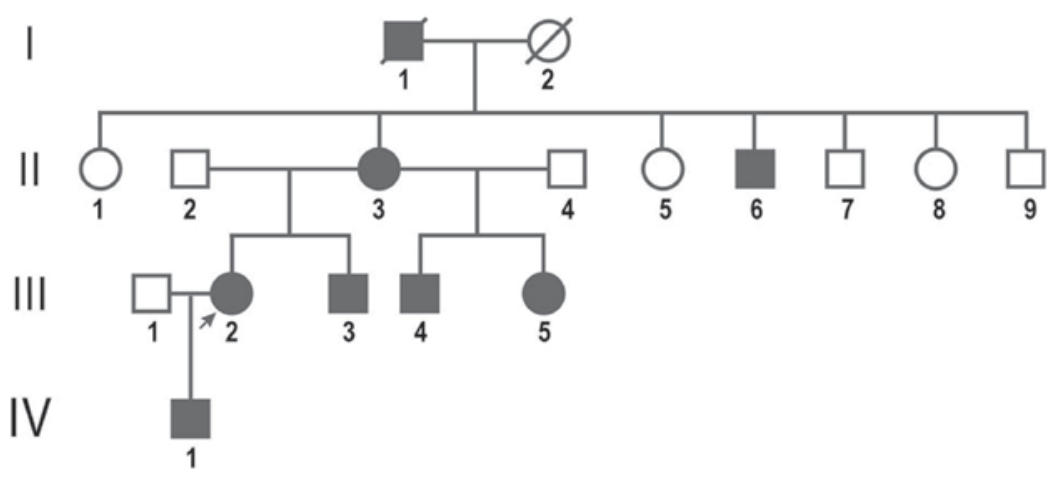

Figure 1. Pedigree of the Charcot-Marie-Tooth disease-affected family. Arrow indicates the proband (III2).

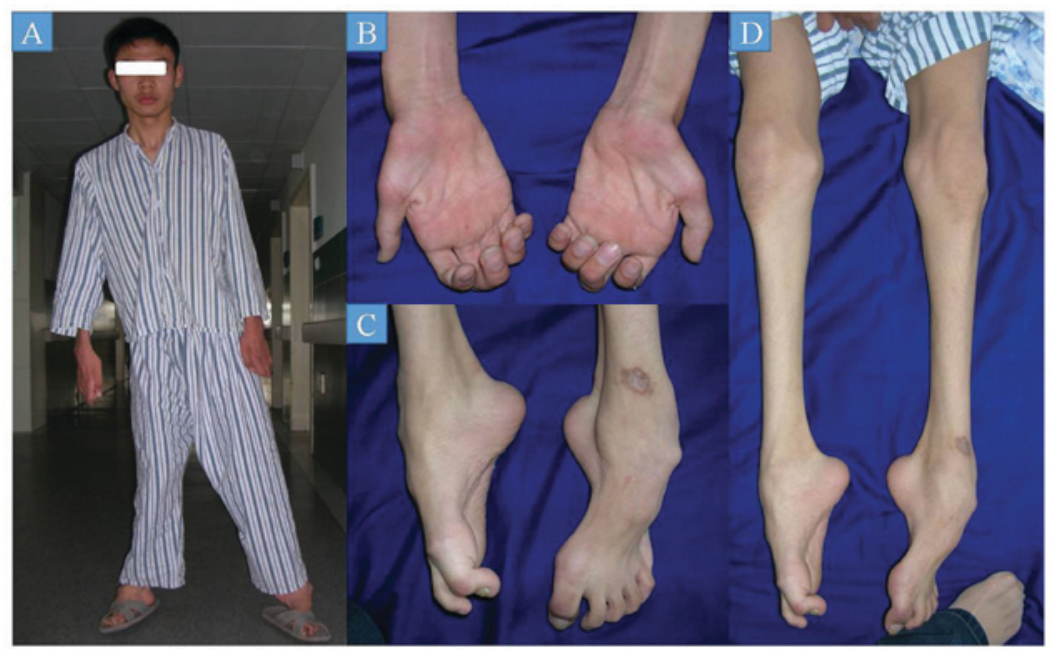

Figure 2. Clinical findings in proband (III2). (A) Unstable walk and abnormal gait. (B) Finger joint contracture. (C) Pes cavus and foot drop. (D) Lower limb weakness.

neighboring residues were exhibited and simulated by PyMOL (Schrödinger, LLC, New York, NY, USA; http://www.pymol. org/). Homology modeling based on the truncated mitofusin-1 (pdb 5GOM, unit 1A) solved with 2.8 A resolution.

\section{Results}

Clinical status of the patients. All 7 affected patients expressed manifestations of bilateral finger contractures and a gait abnormality because of pes cavus, lower limb weakness, foot drop and deformities. The proband (III2) initially presented with symptoms at age 4 years (Fig. 2). Patient (II3) had similar pes cavus, foot muscle atrophy, strephenopodia, and presented with symptoms at age 7 years. Patient (II6) had more critical symptoms, such as lower limb atrophy and limited walking ability after age 30 years with upper limb weakness. The present findings indicated that the clinical manifestations and developmental progression of all affected family members were similar, but the age of initial onset was slightly different, with men aged 3-4 and women 7-8 years. In addition, the male patients exhibited more severe symptoms than the female patients, and they lost their ability to walk independently after age 30 . The electrophysiological examinations were performed on 3 patients (III3, III4, and IV1). The results (recorded in the case history without the electromyogram) exhibited milder
NCV changes. Based on the results of the physical and electrophysiological examinations, the disease of this family was diagnosed as CMT2.

WES identified the candidate mutation in MFN2. The raw data were filtered into clean data and $>96.75 \%$ of reads had $99.9 \%$ accuracy to identify variations among the patients. Following mapping to GRCh37, 99.95\% of the yielded reads were correctly matched, and average sequencing depth reached at least 126-fold. In total, >23,250 exonic and 84,189 intronic SNVs were identified by SAMtools, and highly frequent single nucleotide polymorphism (SNPs) were excluded through international criteria. A similar process was used to screen the 22,013 indels. The candidate variations were produced through three processes: i) Removing highly frequent SNPs, ii) reserving the variations from exons and $10 \mathrm{bp}$ around splicing sites, and iii) eliminating synonymous mutations; eventually 2,223 variations remained. The inheritance model was considered for further advanced analysis to determine the potential disease-causing mutation. In this patient (III2), potential SNVs and indels were separately identified in 541 and 178 candidate genes according to the obvious inheritance pattern of autosomal dominance in this four-generation pedigree. Following several filtering processes, the novel mutation was ultimately identified as c.1190G $>\mathrm{C}$ in exon 10 of MFN2. 


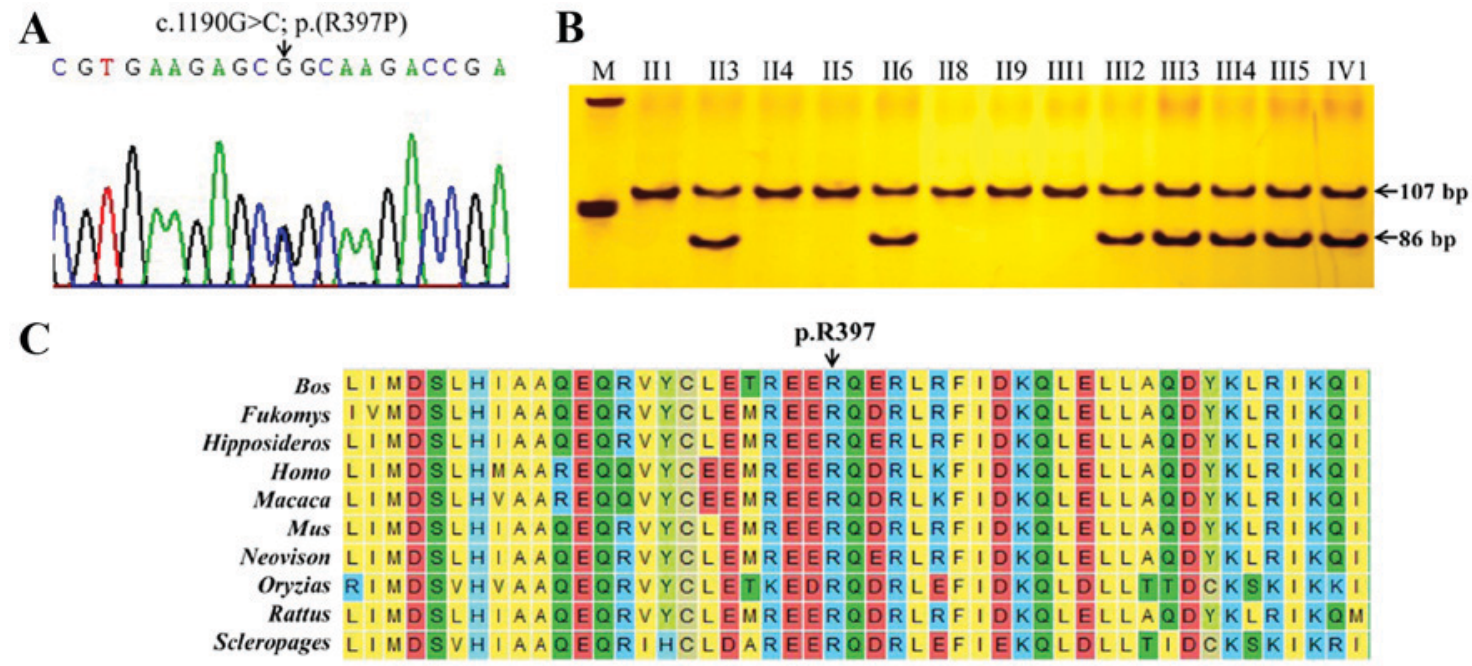

Figure 3. Identification of a missense mutation in the MFN2 gene. (A) The novel mutation c.1190G $>$ C; p.(R397P) was verified by Sanger sequencing (B) PCR-restriction fragment length polymorphism suggested the phenotype and genotype co-segregation in this pedigree. The nested PCR products of the affected family members (II3, II6, III2, III3, III4, III5 and IV1) were digested into two fragments (107 and 86 bp) by MSPI and the others were not affected. (C) Multi-species sequence alignment showing the evolutionarily conserved p.R397 position in MFN2. MFN2, mitofusin 2; PCR, polymerase chain reaction; $\mathrm{M}$, molecular weight standards (50 bp DNA Ladder).
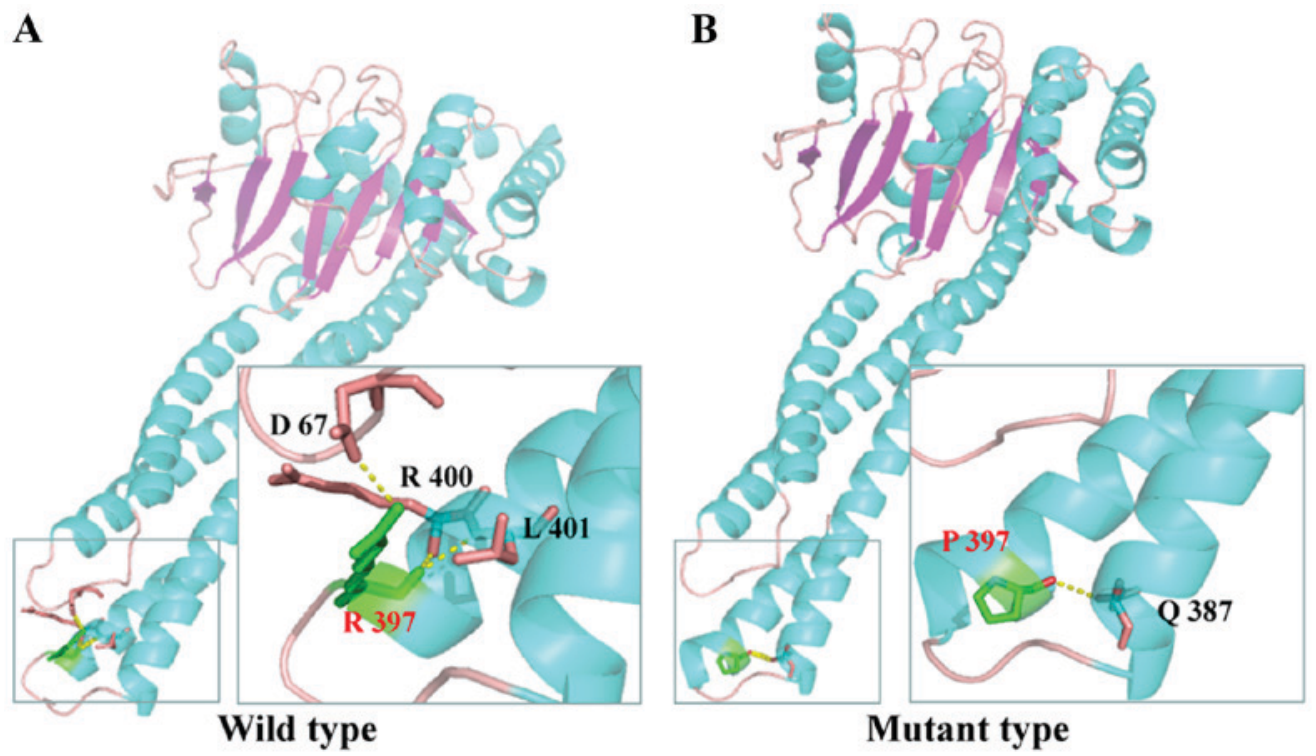

Figure 4. SWISS-MODEL prediction of mitofusin 2 structure and the p.R397P mutation. (A) Amino acid position 397 of wild type is displayed in green. R397 interacted with the R400, L401 and D67 residues through H-bonds and are presented as a yellow dashed line. (B) The formation of H-bonds is predictably altered by the substituted amino acid proline.

This mutation led to substitution of arginine for proline at amino acid residue 397 (p.R397P).

Mutation verification. The candidate mutation in the MFN2 gene was verified by Sanger sequencing. As a result, the variant from Sanger sequencing was completely consistent with that from the WES analysis (Fig. 3A). PCR-RFLP indicated that the wild-type allele failed to be digested by MSPI and the mutant allele was successfully separated into 19 and 86 bp fragments. All affected individuals (II3, II6, III2, III3, III4, III5, and IV1) carrying the heterozygous mutation $(\mathrm{c} .1190 \mathrm{G}>\mathrm{C})$ presented the genotype of three 105, 86 and $19 \mathrm{bp}$ fragments; however, all normal individuals (II1, II4, II5, II8, II9, and III1) and normal controls presented one fragment of $105 \mathrm{bp}$ (Fig. 3B). The close co-segregation of the phenotype and genotype suggested that the R397P variant may be the CMT causative mutation in the present family.

Bioinformatics analysis. A sequence conservation analysis of mitofusin 2 indicated that the amino acid located in position 397 was highly conserved among 10 species (Fig. 3C). Substitution of the wild-type amino acid (arginine) with the mutant amino acid (proline) may change its biological function.

The c.1190G $>$ C; p.(R397P) variation in MFN2 was not present in the HGMD database, indicating that this variation is a rare variation in MFN2. Furthermore, the 
Table I. Prediction of the harmfulness of the c.1190G $>$ C; p. (R397P) mutation in MFN2.

\begin{tabular}{lcl}
\hline Methods & Score & \multicolumn{1}{c}{ Prediction } \\
\hline SIFT & 0.122 & Tolerated \\
Polyphen-2 & 0.548 & Possibly damaging \\
MutationTaster & - & Disease causing \\
M-CAP & 0.1 & Possibly pathogenic \\
\hline
\end{tabular}

SIFT, Scale-invariant feature transform.

SIFT PROVEAN, polyphen-2, Mutation Taster, and M-CAP programs were respectively used to predict the pathogenicity of this variation (Table I). The results from the bioinformatics analysis suggested that c. $1190 \mathrm{G}>\mathrm{C}$ in $M F N 2$ described a disease-causing mutation.

According to the SWISS-MODEL prediction, R397, located in a critical position of the coiled-coil domain, contributed to terminate the helix bundle (Fig. 4A). However, the change in R397P led to extension of the helix bundle. R397 interacted with the R400, L401 and D67 residues through $\mathrm{H}$-bonds, whereas the more hydrophobic residue proline resulted in the reformation of $\mathrm{H}$-bonds with glutamine at position 387 (Fig. 4B).

\section{Discussion}

CMT diseases are a group of clinically and genetically heterogeneous neuropathies with easily confused phenotypes, including neuropathy-associated features and systemic impairment of the central nervous system (13). CMT is divided into two major types based on electrophysiological criteria: Type 1 has slower NCVs $(<38 \mathrm{~m} / \mathrm{s})$, and type 2 has normal or slightly reduced NCVs (14). The present patients exhibited milder changes in the NCVs. As a result, the family was tentatively classified as CMT2. As causative genes associated with CMT are constantly being identified, type 2 has been categorized into several subtypes, making it challenging to determine the correct subtype for a patient with CMT (15).

In the present study, the c.1190G $>C$; p.(R397P) missense mutation in MFN2 was successfully detected by WES, which indicates the power of WES to identify causative mutations for such genetically heterogeneous disorders. Mitofusin 2 encoded by MFN2 is associated with the mobility of mitochondria in peripheral nerves (16-18). A mutation in this protein accounts for $\sim 90 \%$ of severe and early onset CMT2 cases (7). The patients of the current study exhibited onset at a mean age of 5.5 years and expressed the classical CMT2 phenotype, including pes cavus, foot drop and foot muscle atrophy. Those clinical manifestations were highly consistent with the phenotype induced by the MFN2 mutant (5).

These findings suggest that the $\mathrm{c} .1190 \mathrm{G}>\mathrm{C}$ mutation identified in MFN2 is associated with the genetic etiology of the disease in this family for the following reasons: i) This mutation was co-segregated between genotype and phenotype in the family, and absent from all normal controls observed. The inheritance model was consistent with the previously reported autosomal dominant inheritance of CMT2A2A (19). ii) This mutation has no recorded population frequency described in the ExAC Browser and NCBI dbvar database (https://www. ncbi.nlm.nih.gov/dbvar/), indicating that this variation is a rare event in the human genome. iii) The p.R397 amino acid in the mitofusin 2 protein was highly evolutionarily conserved through sequence alignment among 10 species. iv) The substituted amino acid changed the hydrophobicity and charge characteristics of the mitofusin 2 coiled-coiled domain, which may disrupt normal physiological processes. In summary, the novel mutation c.1190G>C; p.(R397P) contributed to the CMT2A phenotype in this four-generation family.

In conclusion, a novel MFN2 mutation was identified in a Chinese family with CMT2A through WES, which expanded the mutational spectrum of CMT2A. Early prenatal intervention is available with an accurate molecular diagnosis of CMT. There are no known effective disease-modifying treatments for MFN2-associated disorders, however, gene-based technologies offer potential to develop treatment, including antisense oligonucleotides, RNA interference to silence mutant expression and induced pluripotent stem cells created in vitro to screen novel drugs (20-22). Further investigation and in vitro and in vivo studies are required to assess the impact of MFN2 mutations on neuronal functions and to further the development of novel therapeutic strategies (23).

\section{Acknowledgements}

Not applicable.

\section{Funding}

The present study was supported by the National Key Research and Development Program of China (grant nos. 2016YFE0128400 and 2016YFC0905100) and CAMS Innovation Fund for Medical Sciences (CIFMS; grant no. 2016-I2M-3-003).

\section{Availability of data and materials}

The datasets used and/or analyzed during the current study are available from the corresponding author on reasonable request.

\section{Authors' contributions}

YY conducted experiments, data analysis and wrote the manuscript. XDW provided pedigree information and performed physical examinations. SL analyzed data. XLZ and $\mathrm{XZ}$ designed and supervised the study. All authors read and approved the final manuscript.

\section{Ethics approval and consent to participate}

Written informed consent was obtained from all participants, and approval was obtained from the Ethics Committee of the Chinese Academy of Medical Sciences (Beijing, China).

\section{Patient consent for publication}

Written consent was obtained from the patients. 


\section{Competing interests}

The authors declare that they have no competing interests.

\section{References}

1. Kazamel M and Boes CJ: Charcot marie tooth disease (CMT): Historical perspectives and evolution. J Neurol 262: 801-805, 2015.

2. Timmerman V, Strickland AV and Züchner S: Genetics of charcot-marie-tooth (CMT) disease within the frame of the human genome project success. Genes (Basel) 5: 13-32, 2014

3. Zuchner S, Mersiyanova IV, Muglia M, Bissar-Tadmouri N, Rochelle J, Dadali EL, Zappia M, Nelis E, Patitucci A, Senderek J, et al: Mutations in the mitochondrial GTPase mitofusin 2 cause Charcot-Marie-Tooth neuropathy type 2A. Nat Genet 36: 449-451, 2004.

4. Tufano M, Cappuccio G, Terrone G, Manganelli F, Pisciotta C, Geroldi A, Capponi S and Del Giudice E: Early onset Charcot-Marie-Tooth neuropathy type $2 \mathrm{~A}$ and severe developmental delay: Expanding the clinical phenotype of MFN2-related neuropathy. J Peripher Nerv Syst 20: 415-418, 2015.

5. Stuppia G, Rizzo F, Riboldi G, Del Bo R, Nizzardo M, Simone C, Comi GP, Bresolin N and Corti S: MFN2-related neuropathies: Clinical features, molecular pathogenesis and therapeutic perspectives. J Neurol Sci 356: 7-18, 2015.

6. Siskind CE, Panchal S, Smith CO, Feely SM, Dalton JC, Schindler AB and Krajewski KM: A review of genetic counseling for Charcot Marie Tooth disease (CMT). J Genet Couns 22: 422-436, 2013.

7. Xie Y, Li X, Liu L, Hu Z, Huang S, Zhan Y, Zi X, Xia K, Tang B and Zhang R: MFN2-related genetic and clinical features in a cohort of Chinese CMT2 patients. J Peripher Nerv Syst 21 $38-44,2016$.

8. Wei X, Ju X, Yi X, Zhu Q, Qu N, Liu T, Chen Y, Jiang H, Yang $\mathrm{G}$, Zhen $\mathrm{R}$, et al: Identification of sequence variants in genetic disease-causing genes using targeted next-generation sequencing. PLoS One 6: e29500, 2011.

9. Wang B, Zheng Z, Wang Z, Zhang X, Yang H, Cai H and Fu Q: A novel missense mutation of TNNI2 in a Chinese family cause distal arthrogryposis type 1. Am J Med Genet A 170A: 135-141, 2016.

10. Jiang Y, Pan J, Guo D, Zhang W, Xie J, Fang Z, Guo C, Fang Q, Jiang W and Guo Y: Two novel mutations in the PPIB gene cause a rare pedigree of osteogenesis imperfecta type IX. Clin Chim Acta 469: 111-118, 2017.

11. Jiang M, Zhao X, Han W, Bian C, Li X, Wang G, Ao Y, Li Y, Yi D, Zhe Y, et al: A novel deletion in TNNI2 causes distal arthrogryposis in a large Chinese family with marked variability of expression. Hum Genet 120: 238-242, 2006.
12. Cao YL, Meng S, Chen Y, Feng JX, Gu DD, Yu B, Li YJ, Yang JY, Liao S, Chan DC and Gao S: MFN1 structures reveal nucleotide-triggered dimerization critical for mitochondrial fusion. Nature 542: 372-376, 2017.

13. Harel T and Lupski JR: Charcot-Marie-Tooth disease and pathways to molecular based therapies. Clin Genet 86: 422-431, 2014.

14. Zimoń M, Battaloğlu E, Parman Y, Erdem S, Baets J, De Vriendt E, Atkinson D, Almeida-Souza L, Deconinck T, Ozes B, et al: Unraveling the genetic landscape of autosomal recessive Charcot-Marie-Tooth neuropathies using a homozygosity mapping approach. Neurogenetics 16: 33-42, 2015.

15. Kostera-Pruszczyk A, Kosinska J, Pollak A, Stawinski P, Walczak A, Wasilewska K, Potulska-Chromik A, Szczudlik P, Kaminska A and Ploski R: Exome sequencing reveals mutations in MFN2 and GDAP1 in severe Charcot-Marie-Tooth disease. J Peripher Nerv Syst 19: 242-245, 2014.

16. Guo X, Chen KH, Guo Y, Liao H, Tang J and Xiao RP: Mitofusin 2 triggers vascular smooth muscle cell apoptosis via mitochondrial death pathway. Circ Res 101: 1113-1122, 2007.

17. Santel A and Fuller MT: Control of mitochondrial morphology by a human mitofusin. J Cell Sci 114: 867-874, 2001

18. Schon K, Spasic-Boskovic O, Brugger K, Graves TD, Abbs S, Park SM, Ambegaonkar G and Armstrong R: Mosaicism for a pathogenic MFN2 mutation causes minimal clinical features of CMT2A in the parent of a severely affected child. Neurogenetics 18: 49-55, 2017.

19. Helbig I, Hodge SE and Ottman R: Familial cosegregation of rare genetic variants with disease in complex disorders. Eur J Hum Genet 21: 444-450, 2013.

20. Deng Y, Wang CC, Choy KW, Du Q, Chen J, Wang Q, Li L, Chung TK and Tang T: Therapeutic potentials of gene silencing by RNA interference: Principles, challenges, and new strategies. Gene 538: 217-227, 2014.

21. Kanasty R, Dorkin JR, Vegas A and Anderson D: Delivery materials for siRNA therapeutics. Nat Mater 12: 967-977, 2013.

22. Nizzardo M, Simone C, Falcone M, Locatelli F, Riboldi G, Comi GP and Corti S: Human motor neuron generation from embryonic stem cells and induced pluripotent stem cells. Cell Mol Life Sci 67: 3837-3847, 2010.

23. Ekins S, Litterman NK, Arnold RJ, Burgess RW, Freundlich JS, Gray SJ, Higgins JJ, Langley B, Willis DE, Notterpek L, et al: A brief review of recent Charcot-Marie-Tooth research and priorities. F1000 Res 4: 53, 2015.

This work is licensed under a Creative Commons Attribution-NonCommercial-NoDerivatives 4.0 International (CC BY-NC-ND 4.0) License. 